\title{
Downregulation of Filamin a Expression in the Aorta Is Correlated With Aortic Dissection
}

\begin{abstract}
Yue Chen ${ }^{1+}$, Xiang Wei ${ }^{1,2,3,4 t}$, Zihao Zhang ${ }^{1}$, Yi He ${ }^{1}$, Bo Huo ${ }^{1}$, Xian Guo ${ }^{1}$, Xin Feng ${ }^{1}$, Ze-Min Fang ${ }^{1}$, Ding-Sheng Jiang ${ }^{1,2,3,4 *}$ and Xue-Hai Zhu ${ }^{1,2,3,4 *}$
\end{abstract}

${ }^{1}$ Division of Cardiothoracic and Vascular Surgery, Sino-Swiss Heart-Lung Transplantation Institute, Tongji Hospital, Tongji Medical College, Huazhong University of Science and Technology, Wuhan, China, ${ }^{2}$ Key Laboratory of Organ Transplantation, Ministry of Education, Chinese Academy of Medical Sciences, Wuhan, China, ${ }^{3}$ NHC Key Laboratory of Organ Transplantation, Chinese Academy of Medical Sciences, Wuhan, China, ${ }^{4}$ Key Laboratory of Organ Transplantation, Chinese Academy of Medical Sciences, Wuhan, China

Filamins (FLNs) are actin cross-linking proteins, and as scaffolding proteins, FLNs are closely associated with the stabilization of the cytoskeleton. Nevertheless, the biological importance of FLNs in aortic dissection (AD) has not been well-elucidated. In this study, we first reanalyzed datasets downloaded from the Gene Expression Omnibus (GEO) database, and we found that in addition to the extracellular matrix, the actin cytoskeleton is a key structure associated with AD. Given that FLNs are involved in remodeling the cytoskeleton to affect cellular functions, we measured their expression levels in the aortas of patients with Stanford type A AD (TAAD). Our results showed that the mRNA and protein levels of FLNA were consistently decreased in dissected aortas of both humans and mice, while the FLNB protein level was upregulated despite decreased FLNB mRNA levels, and comparable expression levels of FLNC were observed between groups. Furthermore, the immunohistochemistry results demonstrated that FLNA was highly expressed in smooth muscle cells (SMCs) of aorta in non-AD samples, and downregulated in the medial layer of the dissected aortas of humans and mice. Moreover, we revealed that FOS and JUN, forming a dimeric transcription factor called AP-1 (activating protein-1), were positively correlated with the expression of FLNA in aorta. Either overexpression of FOS or JUN alone, or overexpression of FOS and JUN together, facilitated the expression of FLNA in primary cultured human aortic SMCs. In the present study, we not only detected the expression pattern of FLNs in aortas of humans and mice with or without $A D$, but we also found that the expression of FLNA in the $A D$ samples was significantly reduced and that AP-1 might regulate the expression of FLNA. Our findings will contribute to the elucidation of the pathological mechanisms of $A D$ and provide potential therapeutic targets for AD.

Keywords: aortic dissection, FLNA, filamins, FOS/JUN, AP-1, bioinformatics

\section{INTRODUCTION}

Aortic dissection (AD) is a life-threatening cardiovascular disorder requiring urgent surgical therapy. Despite advances in diagnostic modalities, surgical treatments, and medical devices, the mortality of AD remains high, and almost $75 \%$ of patients with Stanford type A AD (TAAD) die within 2 weeks when untreated (1). Hence, emergency surgery is currently the optimal solution for 
saving the lives of patients with TAAD. However, the outcome is unpredictable and patients may suffer many complications, such as spinal cord ischemia, stroke, mesenteric ischemia/infarction, and acute renal failure during rapid open surgical repair (2). Therefore, further exploration of the pivotal molecular mechanisms of $\mathrm{AD}$ to identify effective therapeutic targets is urgently needed.

Actins and actin-binding proteins are cytoskeletal proteins that are indispensable for cellular structure and function (3). In the aorta, these structural proteins are closely related to SMC contractile force and play a crucial role in vascular tension (4). Thus, the aberrant expression of these molecules can result in remodeling of the aorta and vascular disorder. For example, mutations in ACTA2, which encodes SMC $\alpha$-actin, are responsible for $14 \%$ of patients with inherited ascending thoracic aortic aneurysms and dissections (5). ACTA2 deficiency in mice reduces elastin levels with increased collagen deposition, facilitating angiotensin II (Ang II)-induced TAAD (6). In addition to actins, smooth muscle 22 (SM22)- $\alpha$, an SMC-specific actin-binding protein, is a contractile marker for SMC phenotype transition (7). The expression of SM22 is decreased in TAAD tissues and the suppression of SM22 in SMCs significantly contributes to proliferation, indicating the transformation from contractile to synthetic SMCs (7). Additionally, the depletion of actin-binding protein Girdin attenuates the proliferation and migration of SMCs to affect vascular remodeling (8). Thus, these studies indicated that actins and actin-binding proteins are vital for the stabilization of SMC functions and vascular homeostasis.

Filamins (FLNs) are actin-filament-crosslinking proteins and consist of three homologous proteins: FLNA, FLNB, and FLNC (9). As actin-binding proteins, FLNs can stabilize delicate threedimensional actin webs and link them to cellular membranes to maintain cellular morphology (9). It was reported that FLNs interact with more than 70 proteins including transmembrane receptors and signaling molecules to play a vital role in cell motility, adhesion, spreading, and signal transduction (10). For SMCs, the degradation of FLNs inhibits differentiation and migration and interrupts phenotype switching (11). FLNA is the most abundant and widely understood actin-binding protein among the three FLN isoforms. Kevin Retailleau et al. reported that the deletion of FLNA in SMCs might cause a reduction in arterial stiffness and a compensatory increase in the conduit artery diameter of mice (12). Furthermore, FLNA is involved with a G protein-coupled P2Y2 nucleotide receptor to regulate the migration of vascular SMCs (13). However, the role of the FLN family in $\mathrm{AD}$ remains unclear.

In the present study, we first analyzed the differentially expressed genes in aortic samples of normal subjects and patients with $\mathrm{AD}$, and further focused on the expression of the FLN family. Compared with non-AD samples, significantly reduced FLNA mRNA and protein levels were observed in the aortic samples obtained from $\mathrm{AD}$ patients. We next generated a murine $\mathrm{AD}$ model by treating mice with $\beta$-aminopropionitrile (BAPN) for 4 weeks and found a similar expression pattern of FLNA in normal and AD mice. Further bioinformatics analysis suggested that the expression levels of FOS and JUN, which form the activator protein-1 (AP-1) complex, were positively correlated with FLNA expression in the aorta of humans. Moreover, both FOS and JUN overexpression can promote the expression of FLNA in cultured primary human aorta smooth muscle cells (HASMCs). Thus, our data indicate that the AP-1/FLNA axis might play a potential role in $\mathrm{AD}$ formation and may serve as a promising therapeutic target for $\mathrm{AD}$.

\section{MATERIALS AND METHODS}

\section{Human Aortic Samples}

All protocols using human specimens were approved by the Human Research Ethics Committees of Tongji Hospital, Tongji Medical College, Huazhong University of Science and Technology, and informed consent was obtained from patients or their family members. Aortic tissues were obtained from patients with TAAD and patients undergoing heart transplantation (controls). All samples were stored in liquid nitrogen or paraformaldehyde as soon as possible after aorta excision to avoid specimen degradation.

\section{Animal Experiments}

All animal experiments were performed in accordance with the protocols approved by the Animal Care and Use Committees of Tongji Hospital, Tongji Medical College, Huazhong University of Science and Technology. All mice (C57BL/6 background) were housed in specific-pathogen-free facilities with a 12 $\mathrm{h}$ light/dark cycle and controlled temperature $\left(20-22^{\circ} \mathrm{C}\right)$. To induce $\mathrm{AD}$, the 3 -week-old mice were stimulated with $0.6 \% \beta$ aminopropionitrile (BAPN, A3134; Sigma-Aldrich) taken orally for 4 weeks. The physical conditions of the mice were observed every day and autopsy was performed when the mice died.

\section{Western Blotting}

Western blotting was performed as previously described (14). Human aorta specimens were homogenized with RIPA lysis buffer containing protease inhibitor complex and phosphatase inhibitors, and the protein concentration was assayed using a BCA protein assay kit (23227, Thermo Fisher Scientific). Twenty micrograms of protein were separated by $10 \%$ sodium dodecyl sulfate-polyacrylamide gel electrophoresis and then transferred to polyvinylidene fluoride membranes (IPVH00010, Millipore). The membranes were blocked with 5\% nonfat milk and then incubated with primary antibodies at $4{ }^{\circ} \mathrm{C}$ overnight. Subsequently, the membranes were washed and incubated with the corresponding horseradish peroxidase-conjugated secondary antibody. Finally, the membranes were incubated in ECL reagents prior to visualization using a ChemiDocTM XRS+ system (Bio-Rad). The antibodies used in this study included FLNA (ab76289, Abcam), FLNB (GTX101206, GeneTex), FLNC (ab180941, Abcam), HA (H3663, Sigma), Flag (F1084, Sigma), and $\beta$-Actin (AC026, ABclonal).

\section{Real-Time PCR}

Real-time PCR was performed using established protocols (15). Total RNA from human aorta specimens was extracted using TRIzol reagent (15596026, Ambion) according to the 
manufacturer's instructions. In total, $5 \mu \mathrm{g}$ of RNA was reversetranscribed using the Transcriptor First Strand cDNA synthesis kit. The relative mRNA levels of FLN family members were detected by a quantitative real-time PCR system using SYBR green (11201ES08, Yeasen), and the results were normalized against $18 \mathrm{~S}$ expression. Primer sequences are as follows: FLNA forward primer 5'-CCGCAATGACAATGACACC-3', FLNA reverse primer $5^{\prime}$-TGGAGATACTGCCACTGAGA-3', FLNB forward primer $5^{\prime}$-ACTGTCATGGCCACAGATGG-3', FLNB reverse primer $5^{\prime}$-AAATCCCAGGCCGTTCATGT-3', FLNC forward primer $5^{\prime}$ - CTCCAGCTACAGCTCCATCC-3', FLNC reverse primer $5^{\prime}$-CCATGTGCTTCACGTACACC- $3^{\prime}$, and $18 \mathrm{~S}$ forward primer $5^{\prime}$-CTCAACACGGGAAACCTCAC-3', $18 \mathrm{~S}$ reverse primer 5'-CGCTCCACCAACTAAGAACG-3' .

\section{Histology and Immunohistochemistry Staining}

Histology and immunohistochemistry staining were performed as previously described (16). Human and mouse aortic samples were fixed in $4 \%$ paraformaldehyde and embedded in paraffin, and then $5-\mu \mathrm{m}$-thick serial sections were stained with hematoxylin-eosin ( $\mathrm{H} \& \mathrm{E})$ for morphological examination. Elastin fibers were visualized using elastic van Gieson (EVG) staining according to the manufacturer's instructions and quantified by counting the total breaks over the length of the vessel. Immunohistochemistry analyses were performed using a standard protocol. The paraffin-embedded sections were incubated with citrate antigen retrieval solution $(\mathrm{pH}$ 6.0, P0083, Beyotime) for antigen retrieval. The sections were incubated with primary antibodies overnight at $4{ }^{\circ} \mathrm{C}$ and then incubated with horseradish peroxidase (HRP)-conjugated secondary antibodies for $1 \mathrm{~h}$. Finally, the DAB horseradish peroxidase color development kit (ZLI-9017, ZSGB-BIO) was used for color development.

\section{Cell Culture}

Primary HASMCs were isolated from the aortas of patients who underwent heart transplantation as previously described (14). Briefly, aortas were stored in DME/F12 medium (SH30023.01; HyClone) at $4^{\circ} \mathrm{C}$, and then the intima and adventitia were stripped under microscope. Furthermore, the medial layer was peeled as thin as possible and minced in a sterile culture flask. Small pieces were placed in the wall of a culture flask without culture medium for $30 \mathrm{~min}$ to enable their adherence to the flask. Next, DME/F12 medium containing 10\% fetal bovine serum (FBS, SH30084.03; HyClone) and 1\% penicillin-streptomycin (15140-122; Thermo Fisher Scientific) was slowly added to the culture flask, which was maintained in a humidified environment at $37^{\circ} \mathrm{C}$ with $5 \% \mathrm{CO}_{2}$. A week later, HASMCs were removed from tissues and then transferred to new culture dishes as first generation. After two passages of growth, the third generation SMCs were used for the cell experiments.

\section{Plasmid Constructs and Cell Treatments}

The full-length coding sequences of the human FOS and JUN genes were amplified from cDNA and then cloned into the pHAGE vector as pHAGE-FOS-HA and pHAGE-JUN-Flag, respectively. pHAGE-FOS-HA and pHAGE-JUN-Flag plasmids, and the packaging plasmids psPAX2 (12260, Addgene) and pMD2.G (12259, Addgene) were cotransfected into HEK293T cells in the presence of polyethylenimine (764604, SigmaAldrich) and incubated for $48 \mathrm{~h}$ and the supernatants containing lentivirus were harvested and filtered through a $0.22 \mu \mathrm{m}$ filter (SLGP033RB, Millipore) for cell infection. HASMCs were infected with the corresponding lentivirus (control, lenti-FOSHA, lenti-JUN-Flag, lenti-FOS-HA+ lenti-JUN-Flag) for $24 \mathrm{~h}$ via hexadimethrine bromide ( $10 \mu \mathrm{g} / \mathrm{mL}, \mathrm{H} 9268$, Sigma-Aldrich) and then cultured with DMEM/F12 medium without FBS for cell synchronization. Finally, the infected cells were maintained in DMEM/F12 medium containing FBS for $24 \mathrm{~h}$ and then collected for subsequent research.

\section{Bioinformatic Analysis}

To obtain the mRNA expression profiles of aortas with or without $\mathrm{AD}$ in humans, the keywords "AD" and "Homo sapiens" were applied to search datasets in the Gene Expression Omnibus (GEO) database (https://www.ncbi.nlm.nih.gov/geo/). In these results, we further analyzed each dataset and excluded all non-mRNA datasets, such as non-coding RNA and DNA methylation profile sets. After assessing all the datasets independently, we included four gene expression datasets "GSE153434," “GSE98770," “GSE52093," and "GSE147026," which are datasets of expression profile array or mRNA high-throughput sequencing of aorta with or without $\mathrm{AD}$ in humans. To analyze the data, the raw expression values of each dataset were normalized by using the "limma" package in $\mathrm{R}$, and $\log 2$ conversion was completed. After processing, differentially expressed genes (DEGs) were filtered based on the criteria adjust.p.value $($ FDR $)<0.05$ and $\log F C>1.5$. The DEGs obtained in each dataset were further analyzed by the $\mathrm{R}$ package "clusterProfiler" for GO, KEGG, and GSE analysis (17). Each term was represented as a circle node, in which color represented its adjusted $p$-value. In addition, the node sizes indicated gene ratios and the mean proportions of genes enriched in the GO or KEGG categories.

To explore the transcription factors regulating FLNA, we queried the database of the website signaling pathways project (https://www.signalingpathways.org/index.jsf) and combined the results with the GSE153434 database to analyze the correlation between the expression of transcription factors and FLNA. The Spearman correlation coefficient was used in the correlation analysis, and $t$-tests were carried out to determine the significant differences.

\section{Statistical Analysis}

The data are presented as the mean \pm standard deviation (SD). All statistical analyses in this study were performed by using SPSS software (version 23.0). Comparisons of the means between 2 groups were performed by independent sample $t$-test and oneway ANOVA was performed for the difference assessments of more than two groups. Correlation analyses were performed using Pearson's correlation analysis. A value of $p<0.05$ was considered statistically significant. 


\section{RESULTS}

\section{Bioinformatics Analysis of mRNA Expression Profiles in Human Aortas}

To explore the differential gene expression in normal controls and patients with TAAD, we reanalyzed four gene expression datasets-GSE147026, GSE153434, GSE52093, and GSE98770downloaded from the GEO database. In terms of biological process, extracellular structure organization was enriched in these four datasets (Figure 1A). Similarly, cellular component and molecular function analysis revealed that extracellular matrix (ECM) was the most highly enriched GO term (Figures 1B,C), consistent with previous investigations (18). In addition to the ECM, actin associated elements were also key factors used to elucidate the pathological process of $\mathrm{AD}$ (Figures 1A-C), such as actin filaments and actin-binding proteins. Furthermore, the KEGG pathway enrichment analysis displayed conspicuous differences in actin cytoskeleton pathways between control and TAAD samples (Figure 1D). Notably, the inflammatory response, which participates in SMC death and ECM degradation, was identified as a common TAAD associated cause in the four datasets according to gene set enrichment analysis (GSEA) (Figures 2A-D). Moreover, the actin cytoskeleton was enriched again by using the $\mathrm{C} 5$ collection (ontology gene sets) used for the GSEA (Figures 2E-H). Thus, these results indicated that abnormalities in the actin cytoskeleton may be involved in the development of TAAD in humans.

\section{The mRNA Expression of FLNA and FLNB Is Downregulated in Patients With TAAD}

Given that the actin cytoskeleton was enriched and because FLN family members are actin-binding proteins, we hypothesized that FLN family members may be involved in the pathogenesis of AD. Therefore, we first analyzed the expression levels of FLNs in the GSE153434 dataset. The results showed that FLNA and FLNB expression was decreased in the aorta of the AD patients, whereas FLNC displayed no difference between the two groups (Figures 3A-C). To validate the results of the dataset analysis, we collected aortic tissues from TAAD patients and non-AD aortas from patients who underwent heart transplantation. In this research, all enrolled TAAD patients were diagnosed through CT angiography (CTA), and true/false lumens were clearly visible in the CTA images (Figure 3D). Significant destructive ECM remodeling of the ascending aorta in $\mathrm{AD}$ identified according to its morphology (Figure 3E). Compared with normal aorta tissues, TAAD aorta tissues showed disorganized elastin fibers with increased fragmentation as indicated by elastin van Gieson (EVG) staining (Figure 3F). We further detected the mRNA levels of FLNs in the aorta of non-AD and TAAD patients by real-time PCR and found lower mRNA levels of FLNA and FLNB in patients with TAAD than in control subjects (Figures $\mathbf{3 G}, \mathbf{H}$ ). However, comparable mRNA levels of FLNC were observed between the two groups (Figure 3I).

Since dilatation of the aorta is one of the characteristics of $\mathrm{AD}$ patients, we next investigated the relationship between the mRNA expression of FLNs and aortic diameters. As shown in
Figures 4A-C, there was no remarkable correlation between the FLN mRNA levels and the diameters of aorta, including the ascending, the thoracic and abdominal aorta, or aortic arch. In contrast, the mRNA expression of FLNA and FLNB was positively correlated in the aorta $(r=0.6, p<0.001)$, but only a weak correlation was found between FLNA and FLNC and between FLNB and FLNC (Figure 4D). Thus, considering the similar expression level of FLNC between the non-AD and AD samples, FLNC might not be involved in the process of $\mathrm{AD}$ and should not be further explored in future research. Together, these results suggested that FLNA and FLNB might be involved in the development of AD.

\section{The Protein Levels of FLNA and FLNB in TAAD Patients}

We further investigated whether the protein level of FLNA was altered during the process of $\mathrm{AD}$. Total protein was extracted from aorta with or without TAAD, and the protein levels of FLNA and FLNB were detected by Western blotting. The results showed that consistent with changes in mRNA levels, the protein level of FLNA also obviously decreased in the TAAD samples compared with that in the controls (Figures 5A,B). Similarly, the results of immunohistochemistry showed that FLNA was highly expressed in the normal aorta but significantly reduced in the aorta of patients with TAAD (Figures 5D,E). However, in contrast to the decrease in mRNA level, the protein level of FLNB significantly increased in the aorta of patients with TAAD, and the results of immunohistochemistry further verified this result (Figures 5A,C,D,F). Thus, these results further indicated that FLNA is a candidate gene affecting $\mathrm{AD}$ development.

\section{The Mouse AD Model Was Successfully Established With Young Mice Through the Administration of BAPN}

To examine the roles of the FLN family in vivo, BAPN was used to generate $\mathrm{AD}$ models in young mice (19). First, we treated 3week-old male mice with $0.6 \%$ BAPN per os for 4 weeks and monitored the condition of the mice daily. We found that after 2 weeks of BAPN treatment, the mice began to die because of dissection rupture. The mortality of BAPN-treated mice after 4 weeks was 15\% (Figure 6A) and the morbidity of AD was $45 \%$ according to macroscopic autopsy (Figures 6B,C). Dissection mainly occurred in the ascending aorta, aorta arch, and thoracic aorta (Figure 6C). Furthermore, the aorta of the dissected mice had pathological changes similar to those of the human aorta, including destructive ECM remodeling and disruption of elastic fibers (Figures 6D,E). Consistently, H\&E staining showed an intimal tear between the true and false lumens, and displayed inflammatory cell infiltration in the false lumen (Figure 6D). According to the degree of elastic fiber fragmentation in the aorta $(0,0-25 \%, 25-50 \%, 50-75 \%$, and $75-100 \%)$, we divided the medial degeneration of the aorta into 5 levels $(0,1,2,3$, and 4) (20). After 4 weeks of BAPN treatment, aortas of 3 mice were almost normal, and aortas of the remaining mice exhibited elastic fiber breakage to varying degrees (Figure 6F). In conclusion, we 

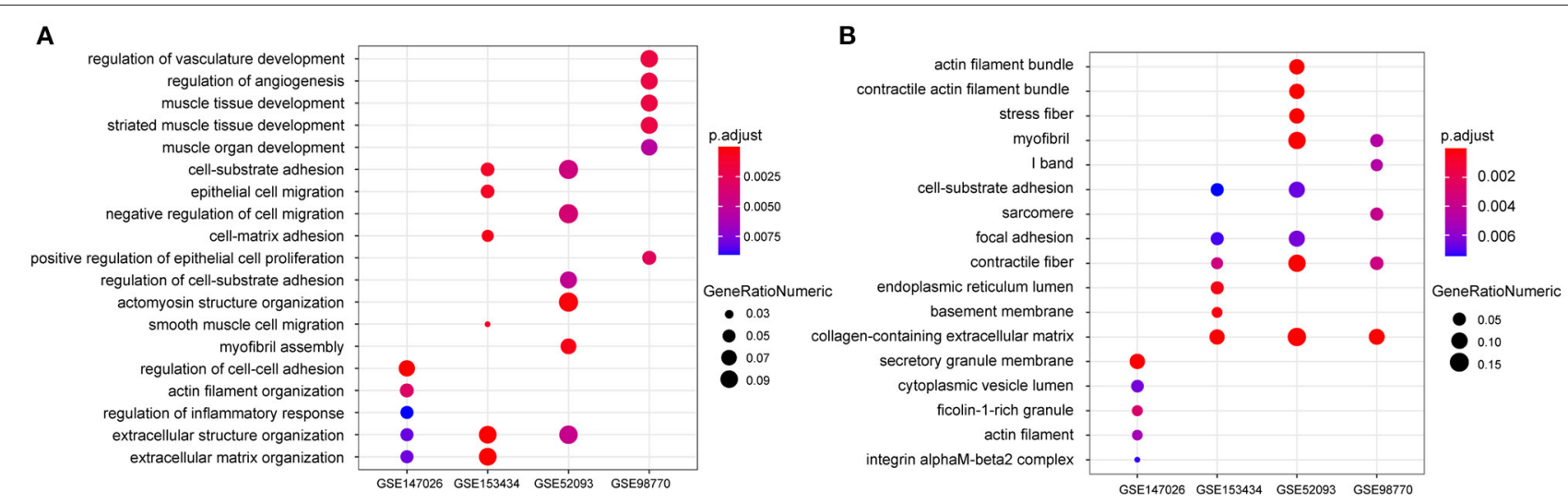

C

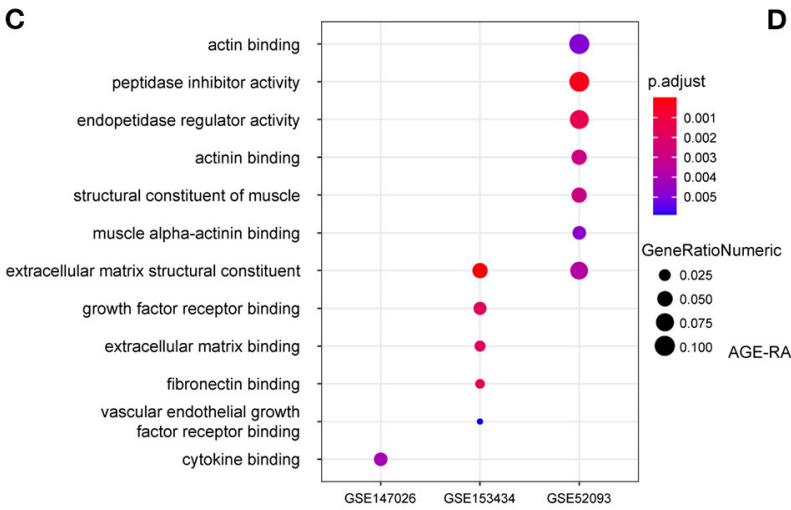

D

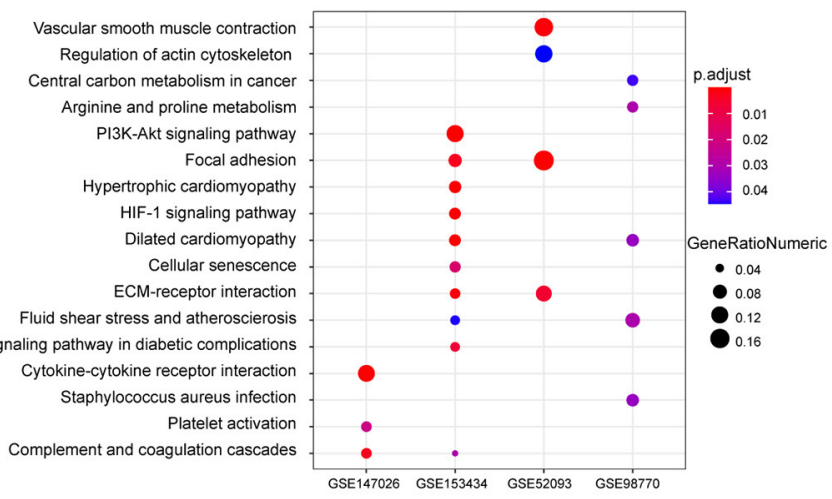

FIGURE 1 | The enrichment analysis shows the differentially expressed genes between the AD and normal groups. (A-C) GO analysis showing the differences of mRNA expression in the aorta between $A D$ and normal groups on biological processes (A) cellular component (B) or molecular function (C) among the four GEO datasets GSE147026, GSE153434, GSE52093, and GSE98770. (D) KEGG enrichment analysis showing the dramatically altered pathways in AD among the indicated datasets.

successfully constructed a mouse $\mathrm{AD}$ model that can simulate the occurrence of human $\mathrm{AD}$ by stimulating young mice with BAPN.

\section{FLNA Was Downregulated in the Aortas of Mice Treated With BAPN}

Using a mouse model of AD, we further examined the FLNA and FLNB protein levels via immunohistochemistry. Consistent with our observations in human samples, FLNA was highly expressed in normal aortae, while FLNB was expressed at low levels (Figure 7A). Furthermore, the protein level of FLNA in the aorta of BAPN-treated mice was significantly decreased compared with its expression in controls (Figures 7A,B), while an obvious increase in FLNB was detected in the aorta of mice after treatment with BAPN (Figures 7A,C). Collectively, the expression patterns of FLNA and FLNB in human AD tissue were confirmed in the mice, which suggested that they might be indispensable for the pathogenesis of $\mathrm{AD}$.

\section{AP-1 Facilitates FLNA Expression in HASMCs}

To further explore the upstream mechanism that inhibited FLNA expression during TAAD development, we collected transcription factors that were reported to be able to bind to the FLNA promoter, as indicated by chromatin immunoprecipitation assay-sequence (ChIP-seq) data obtained from The Signaling Pathways Project (https://www.signalingpathways.org). We then screened these transcription factors in the GSE153434 dataset to identify differentially expressed transcription factors that potentially regulate FLNA expression. Our screening results showed that five transcription factors, FOS, JUN, E2F3, LMO3, and PGR, were differentially expressed in the GSE153434 dataset. Among these factors, FOS, JUN, LOM3, and PGR was downregulated in patients with $\mathrm{AD}$ and positively correlated with FLNA, while E2F3 expression was upregulated (Figure 8A). Notably, FOS and JUN are both subunits of the transcription factor AP-1, which is essential for the function of VSMCs and associated with $\mathrm{AD}$ (21). Therefore, we were very interested to know whether AP-1 is a transcription factor that regulates FLNA expression. Therefore, we overexpressed FOS and JUN in HASMCs via lentivirus infection (Figure 8B). The results showed that either FOS or JUN overexpression in HASMCs promoted FLNA expression, which was enhanced to a greater extent by simultaneous overexpression of FOS and JUN (Figures 8C,D). However, the expression of FLNB was not regulated by FOS or JUN, as evidenced by the comparable expression level of FLNB between the indicated groups of HASMCs (Figure 8C). These results indicated that AP-1 might be the transcription factor regulating FLNA expression during $\mathrm{AD}$ occurrence. 


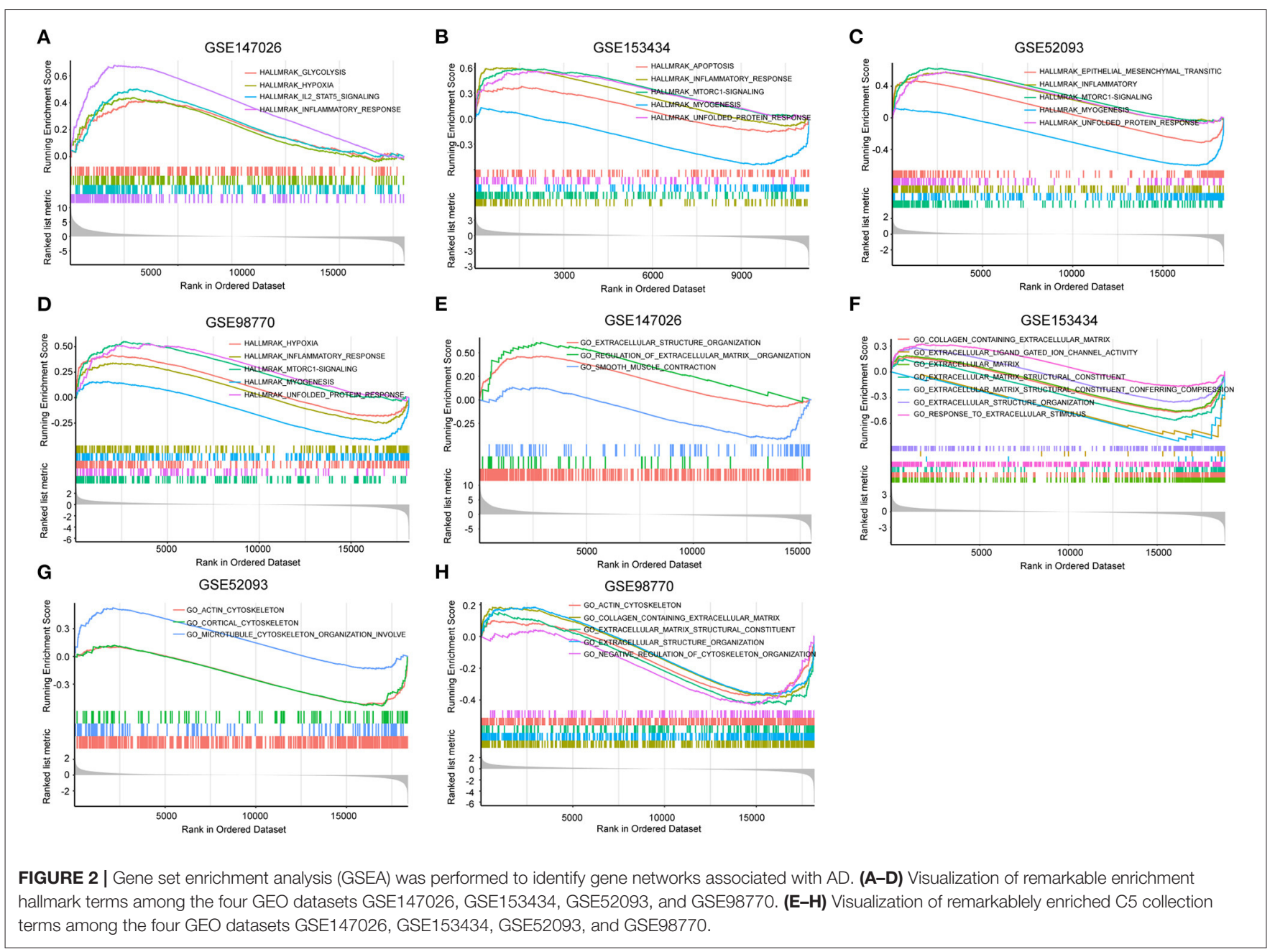

\section{DISCUSSION}

In this study, by reanalyzing datasets in the GEO database, we identified the actin cytoskeleton as a key component in $\mathrm{AD}$ development. We further investigated the expression pattern of the FLN family during AD and found that the mRNA and protein expression levels of FLNA were significantly downregulated in the aortic wall of $\mathrm{AD}$ patients compared with those in the control aorta. Furthermore, a similar scenario was observed in the mouse AD model. Thus, it is highly plausible that FLNA participates in the process of AD. Finally, we found that the transcription complex FOS/JUN can promote the expression of FLNA, which indicated the regulatory mechanism of FLNA in AD.

FLNs are important actin crosslinking proteins and their biological function is largely limited to cell migration (9). Heike Roth et al. showed that FLNA can facilitate efficient migration of human neutrophil-like HL-60 cells via reducing activation of myosin-II (22). It was reported that neutrophils egress from marrow and infiltrate into the aortic adventitia in BAPN/Ang II-treated mice and then release matrix metalloproteinases, such as MMP8 and MMP9, resulting in the degradation of the ECM and the progression of AD (23-25). In addition, Ningpu
$\mathrm{Yu}$ et al. showed that $\mathrm{P} 2 \mathrm{Y} 2 \mathrm{R}$ regulated the proliferation and migration of vascular SMCs by binding FLNA (13). It is wellknown that SMCs exhibit two different phenotypes, acting as contractile or synthetic SMCs, and cells with the contractile phenotype, the predominant form in normal aorta, exhibit relatively low migration and proliferation capacity and high contractile capability (26). Several previous studies confirmed that phenotypic transformation of SMCs from the contractile to synthetic type is one of the typical events in the development of $\mathrm{AD}(27,28)$. The increase of migration ability is the embodiment of the synthetic type, accompanied by the secretion of matrix metalloproteinases and a decline in contractile capability (29, 30). Thus, downregulated FLNA expression in AD patients may be involved in the regulation of migration ability, phenotype transformation, and ECM secretion by VSMCs.

Other studies found that some functions of FLNA in addition to migration might be associated with $\mathrm{AD}$. In the patients with Ehlers-Danlos syndrome and periventricular heterotopia, caused by a loss-of-function mutation in the FLNA gene, was detected and found to contribute the development of aortic dilatation in early adulthood (31). Indeed, the C-terminal fragment of FLNA was previously proposed as a new biomarker of arterial 

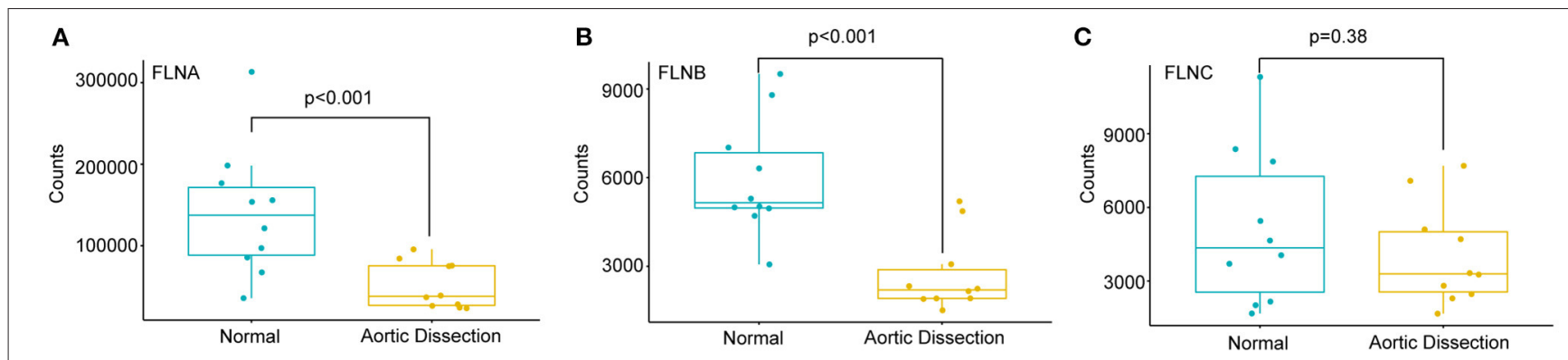

D
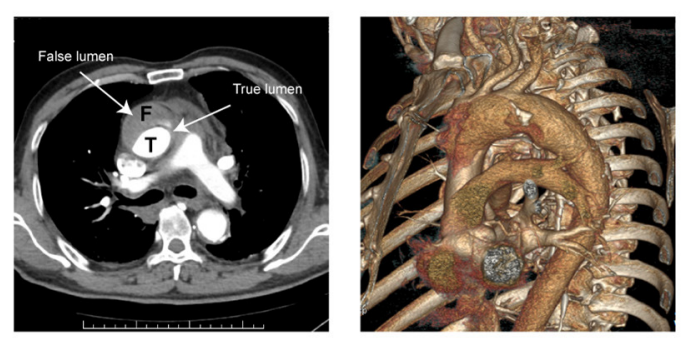

$\mathbf{F}$
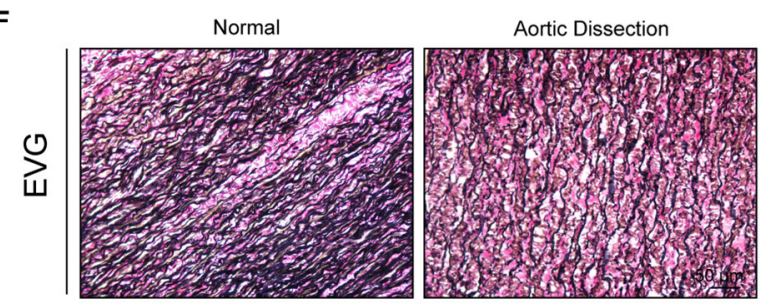

E
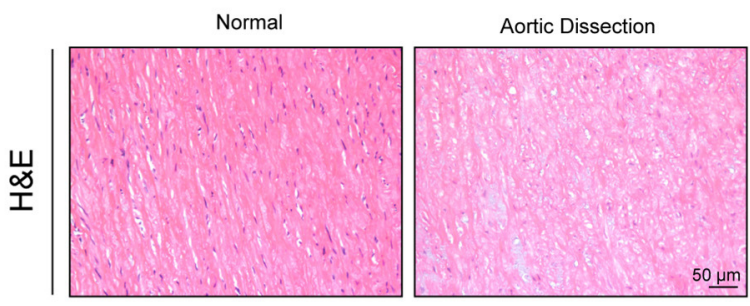

G

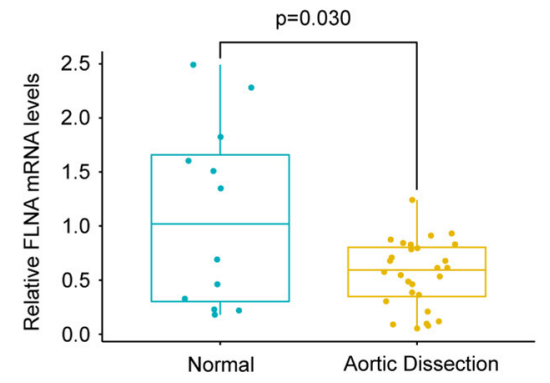

H

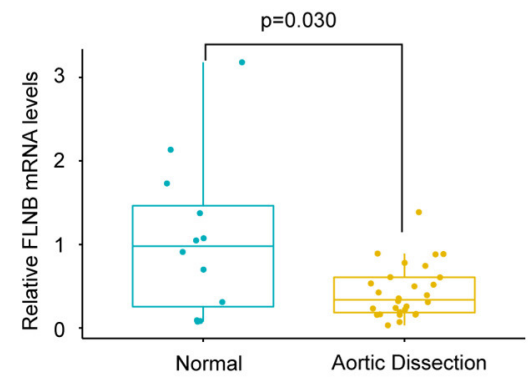

I

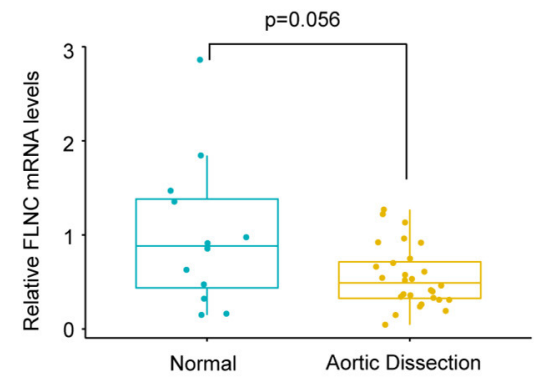

FIGURE 3 | The mRNA levels of FLNA and FLNB were downregulated in human TAAD tissues. (A-C) The expression of FLNA (A) FLNB (B) and FLNC (C) in non-AD and dissected human aortas based on GSE153434 dataset. (D) Representative iterative reconstruction CTA images of TAAD patients, T, true lumen; F, false lumen. (E,F) Representative images of H\&E-stained (E) and EVG-stained (F) aortic sections from non-AD and dissected human aortas. (G-I) The relative mRNA levels of FLNA (G) FLNB (H) and FLNC (I) in the aortas of humans from normal donors $(n=14)$ and $\operatorname{AD}(n=33)$ patients.

wall remodeling in hypertension (32), suggesting that FLNA is of crucial importance for vascular structure. Feng et al. demonstrated that FLNA is necessary for cell junctions in vascular development, and FLNA-null mice displayed failure of vascular remodeling, accompanied by coarse and dilated blood vessels (33). Moreover, smooth muscle-specific FLNA knockout in mice only at the adult stage led to prominent vascular abnormalities, including a reduction in arterial stiffness and a compensatory increase in conduit artery diameter (12). In AD, arterial stiffness is increased owing to the deposition of collagen, resulting in the enhanced aorta susceptibility to dissection and rupture (34). Consistent with aortic dilation as one of the most important risk factors for $\mathrm{AD}$, our results in the present study showed that FLNA is decreased in the aortas of AD patients. These findings indicated that reduced FLNA may contribute to aortic dilation and remodeling during AD. Further studies are needed to verify this hypothesis, especially using knockout or overexpression approaches in animal models.

Similar to FLNA, FLNB also participates in the regulation of migration in many cells. FLNB mediates the firm adhesion of leukocytes to the endothelium through interaction with ICAM$1(35)$, resulting in the transendothelial migration of leukocytes. 


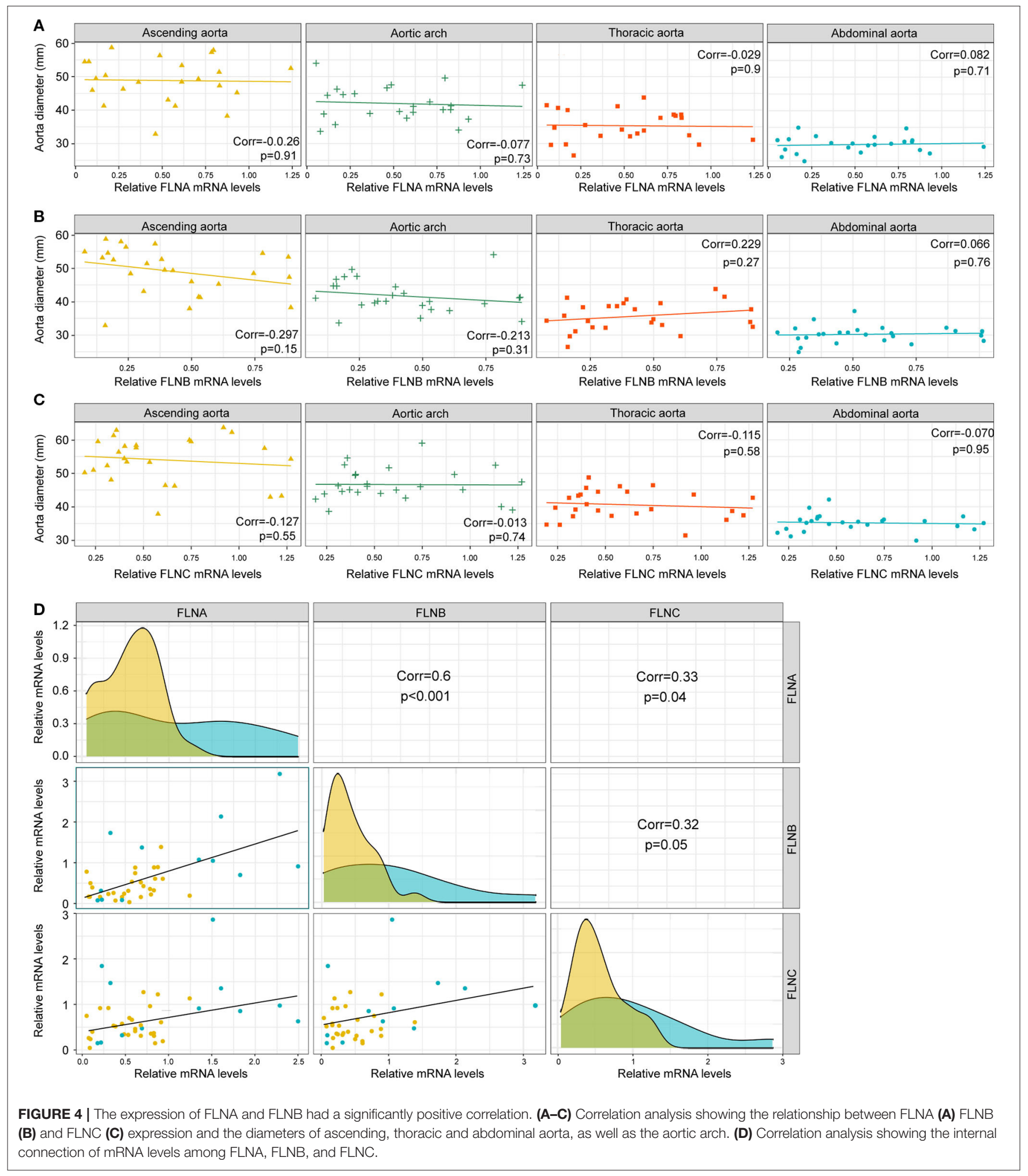

The recruitment of leukocytes to the vessel endothelium results in a local inflammatory response, tears in the intima, and the initiation of $\mathrm{AD}$. In addition, S Bandaru et al. showed that FLNB deficiency enhanced the activity of MMP9 and secretion of vascular endothelial growth factor (VEGF)-A via the RAS/ERK pathway, resulting in tumor growth and metastasis (36). The roles of MMP9 and VEGF have been exhaustively studied in patients with AD. MMP9 degrades type IV collagen, 


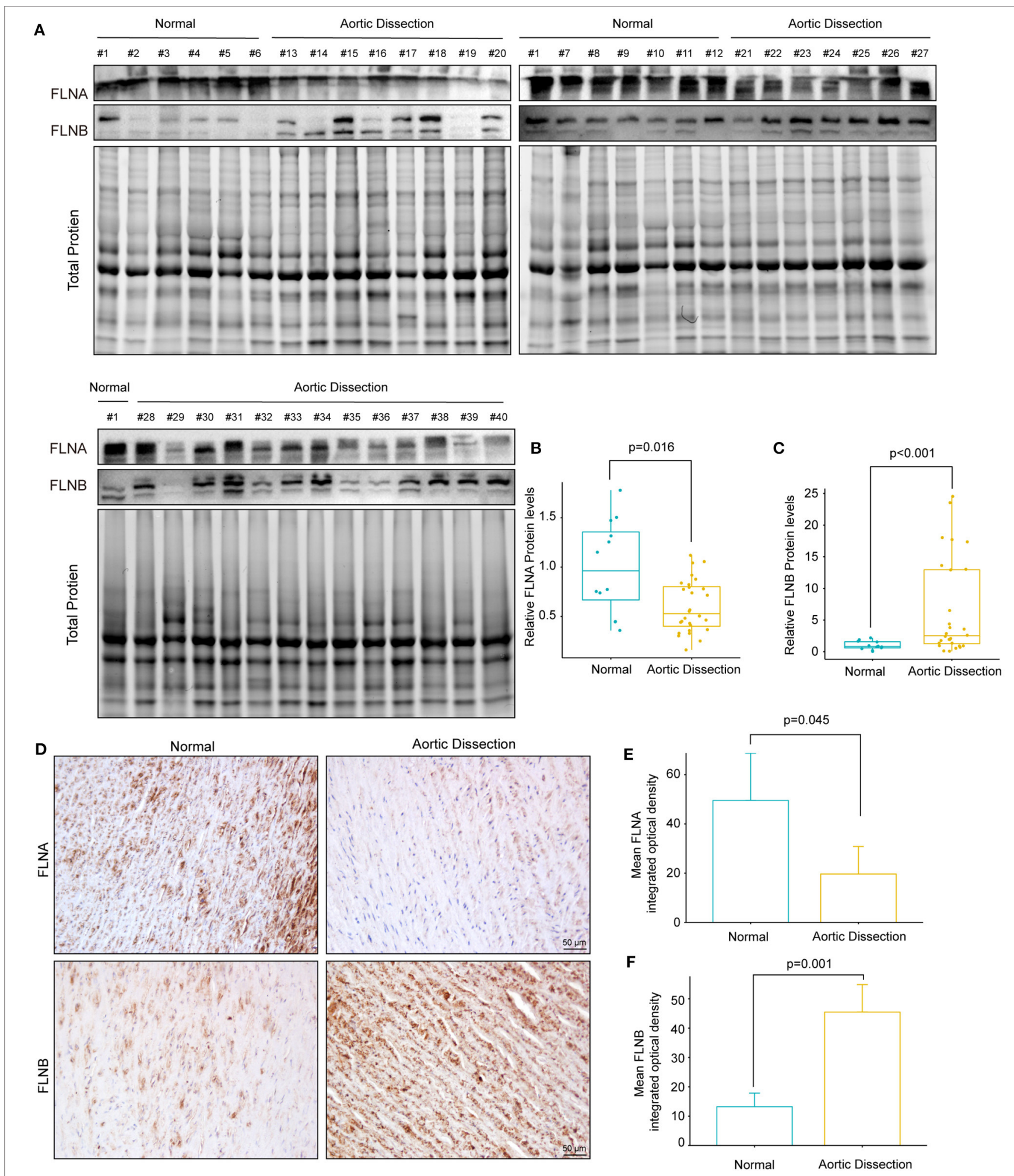

FIGURE 5 | The protein expression of FLNA and FLNB was completely opposite in human TAAD tissues. (A-C) Western blotting and quantitative results of FLNA and FLNB in aortic samples obtained from normal donors $(n=12)$ and $A D(n=28)$ patients. (D-F) Representative images of immunohistochemical staining and quantitative results of FLNA and FLNB in the aortic sections of normal and AD patients $(n=3)$. 
A

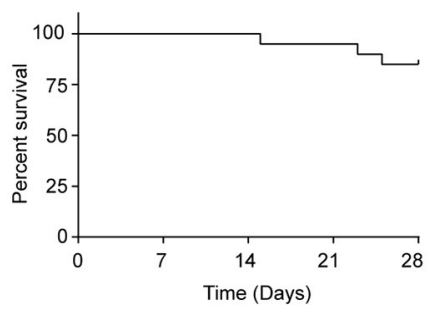

B

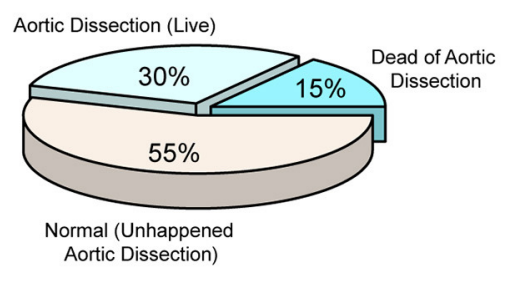

C

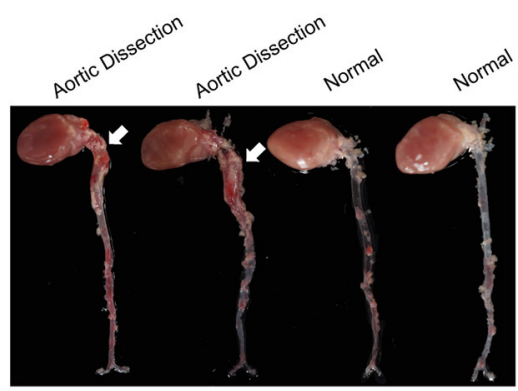

D

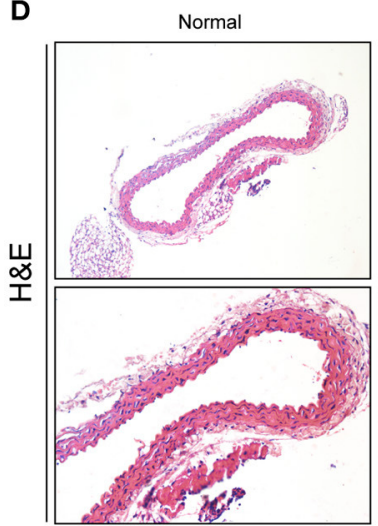

Aortic Dissection

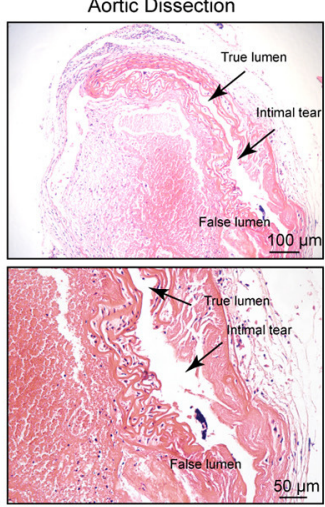

E

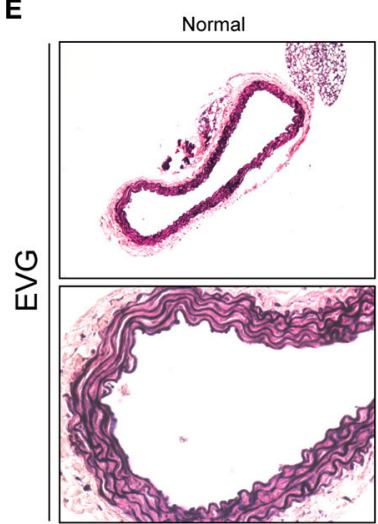

$\mathbf{F}$

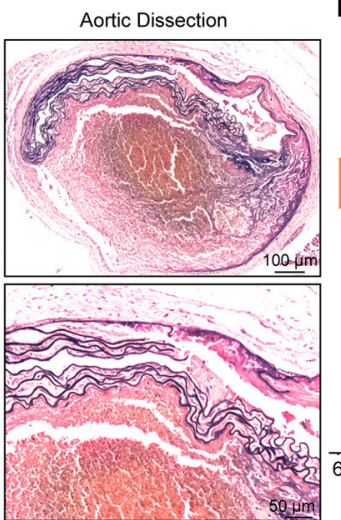

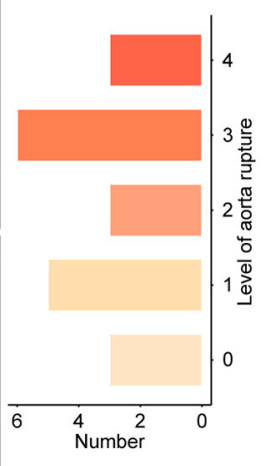

FIGURE 6 | The AD model was successfully established with young mice by administering BAPN. (A) Survival curves of the male mice treated with 0.6\% $\beta$-aminopropionitrile (BAPN) when 3 weeks old. (B) The overall incidences of indicated conditions in mice after 4 weeks of BAPN treatment. (C) Representative macroscopic images of excised aortas after 4 weeks of BAPN treatment. (D,E) Representative images of H\&E-stained (D) and EVG-stained (E) aorta sections from non-AD and dissected mouse aortae. The true/false lumens and intimal tears were labeled in the dissected aorta. (F) Distribution of the degree of elastin rupture in mice treated with BAPN treatment. $n=20$.

elastin, and various basement membrane proteins of SMCs (26), while VEGF contributes to the proinflammatory actions and neoangiogenesis process in aortic wall remodeling (37). It is surprising that the mRNA level of FLNB was decreased, whereas the protein expression increased. The inconsistency between the mRNA and protein levels of FLNB might be attributed to the following causes. First, mRNA posttranscriptional modification is a familiar form of mRNA stability and translation regulation, such as N6-methyladenosine $\left(\mathrm{M}^{6} \mathrm{~A}\right)$ modification (38). For example, ADARB1, an adenosine-to-inosine (A-to-I) RNAediting enzyme, mediates circadian rhythms through mRNA, and plays a role in the posttranscriptional regulation of FLNB (39). In addition, the ubiquitin-proteasome degradation pathway was verified as another mechanism for FLNB post-translational regulation. Heuze et al. reported that ASB2 ubiquitin ligase activity drives proteasome-mediated degradation of the actinbinding protein FLNB and then inhibits cell spreading on fibronectin (40). The E3 ubiquitin ligase specificity subunit ASB $2 \alpha$ regulates cell spreading, migration, and differentiation by interacting with the filamin actin-binding domain, which induces FLNB proteasomal degradation (41). Moreover, a novel ASB2 isoform, ASB2 $\beta$, targets FLNB for proteasomal degradation and impacts myoblast fusion and myotube formation (42). Hence, the ubiquitin proteasome system is a vital pathway for FLNB degradation, and the upregulated FLNB protein level might be attributed to insufficient of degradation in the process of $\mathrm{AD}$, which should be further investigated.

At the mRNA level, we showed that the expression of both FLNA and FLNB was downregulated and that a strong positive correlation between FLNA and FLNB expression was observed in the aortic wall of $\mathrm{AD}$ tissues. This result indicates the possibility that FLNA and FLNB have similar transcriptional regulation mechanisms. Filamin family members have a similar structure and show 70\% amino acid sequence homology (43), suggesting potential functional compensation between FLNA and FLNB. A yeast two-hybrid experiment demonstrated that the FLNB homodimerization domain can strongly interact with the corresponding homologous region of FLNA, and then, via immunoprecipitation assays, FLNA-FLNB heterodimers were verified to exist (44). Previous studies have also demonstrated that FLNA and FLNB play overlapping roles in stabilizing the actin cytoskeleton and cell function (45). In terms of migration, loss of FLNA or FLNB has no effect on migration, but it impairs the initiation of cell migration (46). In chondrocytes, it was demonstrated that loss of FLNA induces upregulation of FLNB expression, and vice versa (47). Taken together with the opposite changes in the protein levels of FLNA and FLNB in $\mathrm{AD}$, it is possible that FLNB upregulation may compensate for the decrease in FLNA. Of course, different and even opposite functions have also been illuminated in other areas 


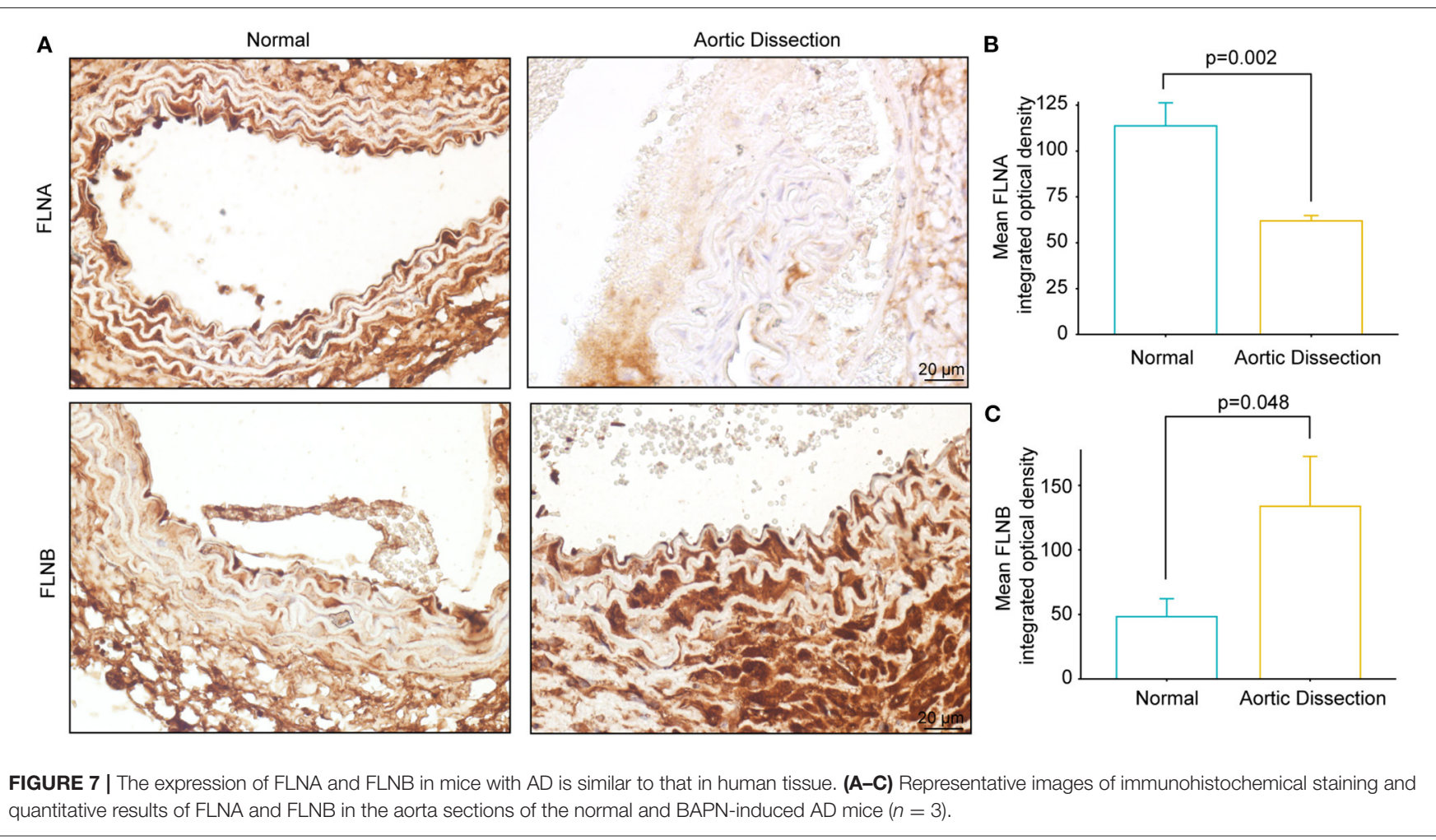

of study. For instance, in RAS-induced lung tumorigenesis, knockout of FLNA significantly reduced the tumor formation and proliferation of fibroblasts via inactivation of ERK and AKT (48). Nevertheless, in contrast to FLNA, FLNB deficiency enhanced RAS-induced tumor growth and metastasis through the RAS/ERK pathway (49).

Another important finding reported here is the transcriptional regulation function of the AP-1 complex (FOS/JUN) on FLNA. To date, few studies have reported the regulatory mechanism of FLNA. Matthew R. Sarkisian et al. revealed that MEKK4 suppression contributes to abnormally high FLNA expression and inhibits neuronal migration (50). In the present study, we revealed that AP-1 might promote FLNA expression in HASMCs. AP-1 is a member of the JUN, FOS, Maf, and ATF subfamilies (51) and well-recognized as a key transcription factor in cell proliferation, death, and oncogenesis (52). It was reported that IL-18 facilitated AP-1-dependent MMP9 transcription, resulting in increased SMC migration ability (53). Investigation into the possible regulation of FLNA by AP-1 suggested that FLNA might be critical for AP-1-mediated SMC migration. Moreover, AP-1 is also a pivotal factor for $\mathrm{AD}$ and regulates medial degeneration (54). Zhang et al. reported that the protein expression of AP-1 in the aorta of $\mathrm{AD}$ was downregulated compared to that in normal aortas, and SIRT1 activated AP-1/decorin signaling to alleviate AD (21). Neutralization of AP-1 via decoy oligodeoxynucleotides repressed aortic elastolysis with reduced fiber breaks and MMP activity in a Marfan syndrome mouse model (54). Therefore, we suspect that the inhibition of AP-1 in SMCs aggravates the development of $\mathrm{AD}$, and we suggest that more investigations are directed to the AP-1/FLNA axis in AD.

In addition to $\mathrm{AD}$, aortic aneurysm is also a vessel disease caused by the medial degeneration. However, there are many differences between dissection and aneurysm. Indeed, there might be aorta dilation before $\mathrm{AD}$ formation, but it would not progress to aneurysm. The rupture of aneurysms is limited, whereas the rupture of $\mathrm{AD}$ shows the formation of true/false lumens, accompanied by extension of the tears. In addition, the most common locations of $\mathrm{AD}$ are the ascending aorta, aorta arch, and thoracic aorta (55), while aneurysms mainly occur on the abdominal aorta (56). Of course, the formation of $\mathrm{AD}$ is accompanied by dilation of the aorta, and aneurysms would develop into dissection and rupture of the aorta. However, it must be emphasized that dissection and aneurysm are two different diseases, not two forms of one disease, which should be distinguished in both clinical treatment and basic research. Currently, CT angiography has widely been applied to $\mathrm{AD}$ diagnosis, and intimal tears and true/false lumens can be clearly distinguished (57).

There are some limitations in this study. First, we detected the FLN family expression in the aortas of mice and patients diagnosed with $\mathrm{AD}$, but functions of FLNs have not been investigated, especially using knockout or overexpression approach in animal models. Second, only the BAPN induced mouse AD model was used to analyze FLNs expression. Two or more mouse models of $\mathrm{AD}$ would make this conclusion more 


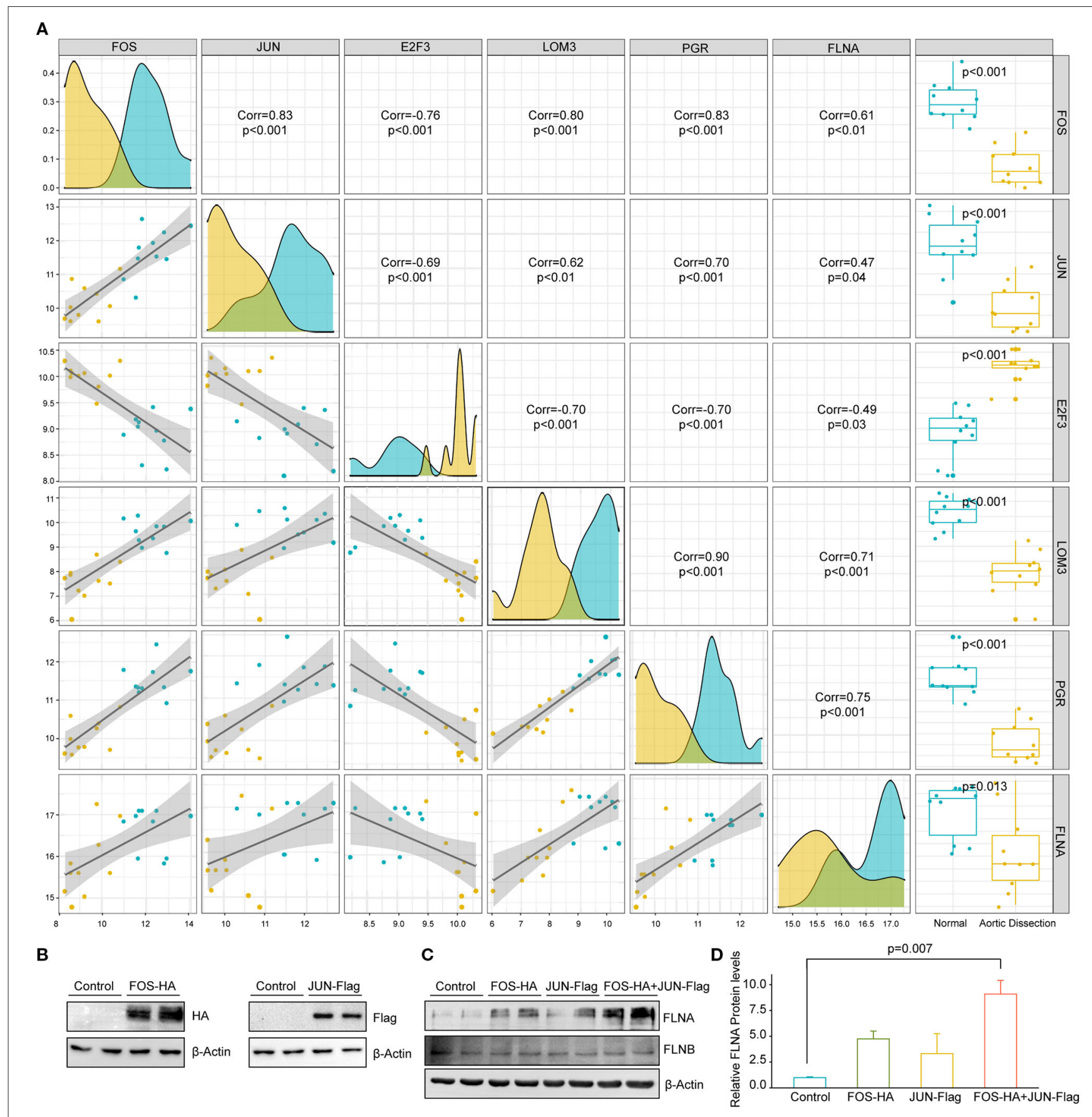

FIGURE 8 | FOS/JUN contributes to FLNA expression in HASMCs. (A) The five transcription factors mostly related to FLNA transcriptional regulation. (B) Western blotting of FOS and JUN expression in HASMCs infected with lenti-FOS-HA and lenti-JUN-Flag. (C,D) Western blot and quantitative results showing FLNA and FLNB expression in HASMCs infected with corresponding lentivirus (Control, FOS-HA, JUN-Flag, FOS-HA+JUN-Flag). $\beta$-Actin served as the loading control.

convincing and meaningful. Finally, we found the possibility of AP-1 on FLNA transcriptional regulation via bioinformatics and further validated that AP-1 could promote FLNA expression in HASMCs. However, further experiments should be done to strengthen this regulation mechanism and to illustrate their functions in $\mathrm{AD}$ development.
In summary, our study demonstrated that the expression level of FLNA was reduced in the aortas of patients with AD, and AP1 might be the transcription factor mediating the expression of FLNA in HASMCs. These results indicated that the AP-1/FLNA axis may be essential for the occurrence of $\mathrm{AD}$, and targeting the AP-1/FLNA axis may be a novel therapeutic strategy for AD. 


\section{DATA AVAILABILITY STATEMENT}

The datasets presented in this study can be found in online repositories. The names of the repository/repositories and accession number(s) can be found in the article/supplementary material.

\section{ETHICS STATEMENT}

The studies involving human participants were reviewed and approved by the Human Research Ethics Committees of Tongji Hospital, Tongji Medical College, Huazhong University of Science and Technology. The patients/participants provided their written informed consent to participate in this study. The animal study was reviewed and approved by the Animal Care and Use Committees of Tongji Hospital, Tongji Medical College, Huazhong University of Science and Technology.

\section{REFERENCES}

1. Cifani N, Proietta M, Tritapepe L, Di Gioia C, Ferri L, Taurino M, et al. Stanford-A acute aortic dissection, inflammation, and metalloproteinases: a review. Ann Med. (2015) 47:441-6. doi: 10.3109/07853890.2015.1073346

2. Erbel R, Aboyans V, Boileau C, Bossone E, Bartolomeo RD, Eggebrecht H, et al. 2014 ESC guidelines on the diagnosis and treatment of aortic diseases: document covering acute and chronic aortic diseases of the thoracic and abdominal aorta of the adult. The task force for the diagnosis and treatment of aortic diseases of the European society of cardiology (ESC). Eur Heart J. (2014) 35:2873-926. doi: 10.1093/eurheartj/ehu281

3. Pollard TD. Actin and actin-binding proteins. Cold Spring Harb Perspect Biol. (2016) 8:a018226. doi: 10.1101/cshperspect.a018226

4. Yamin R, Morgan KG. Deciphering actin cytoskeletal function in the contractile vascular smooth muscle cell. J Physiol. (2012) 590:414554. doi: 10.1113/jphysiol.2012.232306

5. Guo DC, Pannu H, Tran-Fadulu V, Papke CL, Yu RK, Avidan $\mathrm{N}$, et al. Mutations in smooth muscle alpha-actin (ACTA2) lead to thoracic aortic aneurysms and dissections. Nat Genet. (2007) 39:1488-93. doi: 10.1038/ng.2007.6

6. Cheng J, Zhou X, Jiang X, Sun T. Deletion of ACTA2 in mice promotes angiotensin II induced pathogenesis of thoracic aortic aneurysms and dissections. J Thorac Dis. (2018) 10:4733-40. doi: 10.21037/jtd.2018.07.75

7. Han M, Dong LH, Zheng B, Shi JH, Wen JK, Cheng Y. Smooth muscle 22 alpha maintains the differentiated phenotype of vascular smooth muscle cells by inducing filamentous actin bundling. Life Sci. (2009) 84:394401. doi: 10.1016/j.lfs.2008.11.017

8. Miyake H, Maeda K, Asai N, Shibata R, Ichimiya H, Isotani-Sakakibara M, et al. The actin-binding protein girdin and its akt-mediated phosphorylation regulate neointima formation after vascular injury. Circ Res. (2011) 108:11709. doi: 10.1161/CIRCRESAHA.110.236174

9. Stossel TP, Condeelis J, Cooley L, Hartwig JH, Noegel A, Schleicher M, et al. Filamins as integrators of cell mechanics and signalling. Nat Rev Mol Cell Biol. (2001) 2:138-45. doi: 10.1038/35052082

10. Zhou X, Boren J, Akyurek LM. Filamins in cardiovascular development. Trends Cardiovasc Med. (2007) 17:222-9. doi: 10.1016/j.tcm.2007.08.001

11. Zhu G, Chen H, Zhang W. Phenotype switch of vascular smooth muscle cells after siRNA silencing of filamin. Cell Biochem Biophys. (2011) 61:4752. doi: 10.1007/s12013-011-9159-7

12. Retailleau K, Arhatte M, Demolombe S, Jodar M, Baudrie V, Offermanns $\mathrm{S}$, et al. Smooth muscle filamin A is a major determinant of conduit artery structure and function at the adult stage. Pflügers Archiv Eur J Physiol. (2016) 468:1151-60. doi: 10.1007/s00424-016-1813-x

\section{AUTHOR CONTRIBUTIONS}

YC and XW performed the cell and animal experiments of this study and data analysis. $\mathrm{ZZ}$ and $\mathrm{YH}$ participated in bioinformatics analysis. $\mathrm{BH}$ and $\mathrm{XG}$ cultured the primary HASMCs and constructed the plasmid. XF and Z-MF collected the human aorta tissues. X-HZ and D-SJ designed the work and finished the manuscript. All authors contributed to the article and approved the submitted version.

\section{FUNDING}

This work was supported by grants from the National Natural Science Foundation of China (NOs. 82070488, 81873458, and 81974013).

13. Yu N, Erb L, Shivaji R, Weisman GA, Seye CI. Binding of the P2Y2 nucleotide receptor to filamin A regulates migration of vascular smooth muscle cells. Circ Res. (2008) 102:581-8. doi: 10.1161/CIRCRESAHA.107.162271

14. Chen TQ, Hu N, Huo B, Masau JF, Yi X, Zhong XX, et al. EHMT2/G9a inhibits aortic smooth muscle cell death by suppressing autophagy activation. Int J Biol Sci. (2020) 16:1252-63. doi: 10.7150/ijbs.38835

15. Li R, Yi X, Wei X, Huo B, Guo X, Cheng C, et al. EZH2 inhibits autophagic cell death of aortic vascular smooth muscle cells to affect aortic dissection. Cell Death Dis. (2018) 9:180. doi: 10.1038/s41419-017-0213-2

16. Jiang DS, Yi X, Li R, Su YS, Wang J, Chen ML, et al. The histone methyltransferase mixed lineage leukemia (MLL) 3 may play a potential role on clinical dilated cardiomyopathy. Mol Med. (2017) 23:196203. doi: 10.2119/molmed.2017.00012

17. Yu G, Wang L-G, Han Y, He Q-Y. Clusterprofiler: an R package for comparing biological themes among gene clusters. OMICS. (2012) 16:2847. doi: $10.1089 /$ omi.2011.0118

18. Zhang L, Yu C, Chang Q, Luo X, Qiu J, Liu S. Comparison of gene expression profiles in aortic dissection and normal human aortic tissues. Biomed Rep. (2016) 5:421-7. doi: 10.3892/br.2016.740

19. Jiang DS, Yi X, Zhu XH, Wei X. Experimental in vivo and ex vivo models for the study of human aortic dissection: promises and challenges. Am J Transl Res. (2016) 8:5125-40.

20. Schulte S, Sun J, Libby P, Macfarlane L, Sun C, Lopez-Ilasaca M, et al. Cystatin $\mathrm{C}$ deficiency promotes inflammation in angiotensin II-induced abdominal aortic aneurisms in atherosclerotic mice. Am J Pathol. (2010) 177:456-63. doi: 10.2353/ajpath.2010.090381

21. Zhang K, Pan X, Zheng J, Liu Y, Sun L. SIRT1 protects against aortic dissection by regulating AP-1/decorin signaling-mediated PDCD4 activation. Mol Biol Rep. (2020) 47:2149-59. doi: 10.1007/s11033-020-05314-9

22. Roth H, Samereier M, Begandt D, Pick R, Salvermoser M, Brechtefeld D, et al. Filamin A promotes efficient migration and phagocytosis of neutrophil-like HL-60 cells. Eur J Cell Biol. (2017) 96:553-66. doi: 10.1016/j.ejcb.2017.05.004

23. Anzai A, Shimoda M, Endo J, Kohno T, Katsumata Y, Matsuhashi T, et al. Adventitial CXCL1/G-CSF expression in response to acute aortic dissection triggers local neutrophil recruitment and activation leading to aortic rupture. Circ Res. (2015) 116:612-23. doi: 10.1161/CIRCRESAHA.116.304918

24. Wilson WR, Schwalbe EC, Jones JL, Bell PR, Thompson MM. Matrix metalloproteinase 8 (neutrophil collagenase) in the pathogenesis of abdominal aortic aneurysm. Br J Surg. (2005) 92:828-33. doi: 10.1002/bjs.4993

25. Kurihara T, Shimizu-Hirota R, Shimoda M, Adachi T, Shimizu $\mathrm{H}$, Weiss SJ, et al. Neutrophil-derived matrix metalloproteinase 9 triggers acute aortic dissection. Circulation. (2012) 126:307080. doi: 10.1161/CIRCULATIONAHA.112.097097 
26. Wu D, Shen YH, Russell L, Coselli JS, LeMaire SA. Molecular mechanisms of thoracic aortic dissection. J Surg Res. (2013) 184:907-24. doi: 10.1016/j.jss.2013.06.007

27. Wang L, Zhang J, Fu W, Guo D, Jiang J, Wang Y. Association of smooth muscle cell phenotypes with extracellular matrix disorders in thoracic aortic dissection. J Vasc Surg. (2012) 56:1698-709:709 e1. doi: 10.1016/j.jvs.2012.05.084

28. Ailawadi G, Moehle CW, Pei H, Walton SP, Yang Z, Kron IL, et al. Smooth muscle phenotypic modulation is an early event in aortic aneurysms. J Thorac Cardiovasc Surg. (2009) 138:1392-9. doi: 10.1016/j.jtcvs.2009.07.075

29. An Z, Liu Y, Song ZG, Tang H, Yuan Y, Xu ZY. Mechanisms of aortic dissection smooth muscle cell phenotype switch. J Thorac Cardiovasc Surg. (2017) 154:1511-21 e6. doi: 10.1016/j.jtcvs.2017.05.066

30. Fisher SA. Vascular smooth muscle phenotypic diversity and function. Physiol Genom. (2010) 42A:169-87. doi: 10.1152/physiolgenomics.00111.2010

31. Sheen VL, Jansen A, Chen MH, Parrini E, Morgan T, Ravenscroft R, et al. Filamin A mutations cause periventricular heterotopia with ehlers-danlos syndrome. Neurology. (2005) 64:254-62. doi: 10.1212/01.WNL.0000149512.79621.DF

32. Delbosc S, Haloui M, Louedec L, Dupuis M, Cubizolles M, Podust VN, et al. Proteomic analysis permits the identification of new biomarkers of arterial wall remodeling in hypertension. Mol Med. (2008) 14:38394. doi: 10.2119/2008-00030.Delbosc

33. Feng Y, Chen MH, Moskowitz IP, Mendonza AM, Vidali L, Nakamura F, et al. Filamin A (FLNA) is required for cell-cell contact in vascular development and cardiac morphogenesis. Proc Natl Acad Sci USA. (2006) 103:1983641. doi: 10.1073/pnas.0609628104

34. Lyle AN, Raaz U. Killing me unsoftly: causes and mechanisms of arterial stiffness. Arterioscler Thromb Vasc Biol. (2017) 37:e1-11. doi: 10.1161/ATVBAHA.116.308563

35. Kanters E, van Rijssel J, Hensbergen PJ, Hondius D, Mul FP, Deelder AM, et al. Filamin B mediates ICAM-1-driven leukocyte transendothelial migration. J Biol Chem. (2008) 283:31830-9. doi: 10.1074/jbc.M804888200

36. Zhou X, Tian F, Sandzen J, Cao R, Flaberg E, Szekely L, et al. Filamin $\mathrm{B}$ deficiency in mice results in skeletal malformations and impaired microvascular development. Proc Natl Acad Sci USA. (2007) 104:391924. doi: $10.1073 /$ pnas.0608360104

37. Del PF, di Gioia C, Tritapepe L, Ferri L, Leopizzi M, Nofroni I, et al. The multitasking role of macrophages in stanford type A acute aortic dissection. Cardiology. (2014) 127:123-9. doi: 10.1159/000355253

38. Chen J, Wei X, Yi X, Jiang D-S. RNA modification by m6A methylation in cardiovascular disease. Oxid Med Cell Longev. (2021) 2021:8813909. doi: 10.1155/2021/8813909

39. Terajima H, Yoshitane H, Ozaki H, Suzuki Y, Shimba S, Kuroda S, et al. ADARB1 catalyzes circadian A-to-I editing and regulates RNA rhythm. Nat Genet. (2017) 49:146-51. doi: 10.1038/ng.3731

40. Heuze ML, Lamsoul I, Baldassarre M, Lad Y, Leveque S, Razinia Z, et al. ASB2 targets filamins A and B to proteasomal degradation. Blood. (2008) 112:5130-40. doi: 10.1182/blood-2007-12-128744

41. Razinia Z, Baldassarre M, Bouaouina M, Lamsoul I, Lutz PG, Calderwood DA. The E3 ubiquitin ligase specificity subunit ASB2alpha targets filamins for proteasomal degradation by interacting with the filamin actin-binding domain. J Cell Sci. (2011) 124:2631-41. doi: 10.1242/jcs.084343

42. Bello NF, Lamsoul I, Heuzé ML, Métais A, Moreaux G, Calderwood DA, et al. The E3 ubiquitin ligase specificity subunit ASB2 $\beta$ is a novel regulator of muscle differentiation that targets filamin B to proteasomal degradation. Cell Death Differ. (2009) 16:921-32. doi: 10.1038/cdd.2 009.27

43. van der Flier A, Sonnenberg A. Structural and functional aspects of filamins. Biochim Biophys Acta. (2001) 1538:99117. doi: 10.1016/S0167-4889(01)00072-6

44. Sheen VL, Feng Y, Graham D, Takafuta T, Shapiro SS, Walsh CA. Filamin A and Filamin $\mathrm{B}$ are co-expressed within neurons during periods of neuronal migration and can physically interact. Hum Mol Genet. (2002) 11:284554. doi: $10.1093 / \mathrm{hmg} / 11.23 .2845$

45. Nakamura F, Stossel TP, Hartwig JH. The filamins: organizers of cell structure and function. Cell Adh Migr. (2011) 5:160-9. doi: 10.4161/cam.5.2.14401

46. Baldassarre M, Razinia Z, Burande CF, Lamsoul I, Lutz PG, Calderwood DA. Filamins regulate cell spreading and initiation of cell migration. PLoS ONE. (2009) 4:e7830. doi: 10.1371/journal.pone.0007830

47. Hu J, Lu J, Goyal A, Wong T, Lian G, Zhang J, et al. Opposing FlnA and $\mathrm{FlnB}$ interactions regulate RhoA activation in guiding dynamic actin stress fiber formation and cell spreading. Hum Mol Genet. (2017) 26:1294304. doi: $10.1093 / \mathrm{hmg} / \mathrm{ddx} 047$

48. Nallapalli RK, Ibrahim MX, Zhou AX, Bandaru S, Sunkara SN, Redfors B, et al. Targeting filamin A reduces K-RAS-induced lung adenocarcinomas and endothelial response to tumor growth in mice. Mol Cancer. (2012) 11:50. doi: 10.1186/1476-4598-11-50

49. Bandaru S, Zhou AX, Rouhi P, Zhang Y, Bergo MO, Cao Y, et al. Targeting filamin $\mathrm{B}$ induces tumor growth and metastasis via enhanced activity of matrix metalloproteinase-9 and secretion of VEGF-A. Oncogenesis. (2014) 3:e119. doi: 10.1038/oncsis.2014.33

50. Yuan Y, Wang C, Xu J, Tao J, Xu Z, Huang S. BRG1 overexpression in smooth muscle cells promotes the development of thoracic aortic dissection. BMC Cardiovasc Disord. (2014) 14:144. doi: 10.1186/1471-2261-14-144

51. Shaulian E, Karin M. AP-1 as a regulator of cell life and death. Nat Cell Biol. (2002) 4:E131-6. doi: 10.1038/ncb0502-e131

52. Tewari D, Nabavi SF, Nabavi SM, Sureda A, Farooqi AA, Atanasov AG, et al. Targeting activator protein 1 signaling pathway by bioactive natural agents: possible therapeutic strategy for cancer prevention and intervention. Pharmacol Res. (2018) 128:366-75. doi: 10.1016/j.phrs.2017.09.014

53. Chandrasekar B, Mummidi S, Mahimainathan L, Patel DN, Bailey SR, Imam SZ, et al. Interleukin-18-induced human coronary artery smooth muscle cell migration is dependent on NF-кB- and AP-1-mediated matrix metalloproteinase- 9 expression and is inhibited by atorvastatin. J Biol Chem. (2006) 281:15099-109. doi: 10.1074/jbc.M600200200

54. Arif R, Zaradzki M, Remes A, Seppelt P, Kunze R, Schroder H, et al. AP-1 oligodeoxynucleotides reduce aortic elastolysis in a murine model of marfan syndrome. Mol Ther Nucleic Acids. (2017) 9:6979. doi: 10.1016/j.omtn.2017.08.014

55. Golledge J, Eagle KA. Acute aortic dissection. Lancet. (2008) 372:5566. doi: 10.1016/S0140-6736(08)60994-0

56. Davis FM, Daugherty A, Lu HS. Updates of recent aortic aneurysm research. Arterioscler Thromb Vasc Biol. (2019) 39:e83-90. doi: 10.1161/ATVBAHA.119.312000

57. Nienaber CA, Clough RE. Management of acute aortic dissection. LANCET. (2015) 385:800-11. doi: 10.1016/S0140-6736(14)61005-9

Conflict of Interest: The authors declare that the research was conducted in the absence of any commercial or financial relationships that could be construed as a potential conflict of interest.

Publisher's Note: All claims expressed in this article are solely those of the authors and do not necessarily represent those of their affiliated organizations, or those of the publisher, the editors and the reviewers. Any product that may be evaluated in this article, or claim that may be made by its manufacturer, is not guaranteed or endorsed by the publisher.

Copyright (c) 2021 Chen, Wei, Zhang, He, Huo, Guo, Feng, Fang, Jiang and Zhu. This is an open-access article distributed under the terms of the Creative Commons Attribution License (CC BY). The use, distribution or reproduction in other forums is permitted, provided the original author(s) and the copyright owner(s) are credited and that the original publication in this journal is cited, in accordance with accepted academic practice. No use, distribution or reproduction is permitted which does not comply with these terms. 